Article

\title{
Hybrid Weighted Arithmetic and Geometric Aggregation Operator of Neutrosophic Cubic Sets for MADM
}

\author{
Lilian Shi $*$ (D) and Yue Yuan \\ Department of Electrical Automation, Shaoxing University, 508 Huancheng West Road, Shaoxing 312000, China; \\ 15733719615@163.com \\ * Correspondence: cssll@usx.edu.cn
}

Received: 4 January 2019; Accepted: 19 February 2019; Published: 21 February 2019

check for updates

\begin{abstract}
Neutrosophic cubic sets (NCSs) can express complex multi-attribute decision-making (MADM) problems with its interval and single-valued neutrosophic numbers simultaneously. The weighted arithmetic average (WAA) and geometric average (WGA) operators are common aggregation operators for handling MADM problems. However, the neutrosophic cubic weighted arithmetic average (NCWAA) and neutrosophic cubic geometric weighted average (NCWGA) operators may result in some unreasonable aggregated values in some cases. In order to overcome the drawbacks of the NCWAA and NCWGA, this paper developed a new neutrosophic cubic hybrid weighted arithmetic and geometric aggregation (NCHWAGA) operator and investigates its suitability and effectiveness. Then, we established a MADM method based on the NCHWAGA operator. Finally, a MADM problem with neutrosophic cubic information was provided to illustrate the application and effectiveness of the proposed method.
\end{abstract}

Keywords: weighted geometric operator; weighted average operator; neutrosophic cubic sets; multi-attribute decision-making (MADM); neutrosophic cubic hybrid weighted arithmetic and geometric aggregation operator (NCHWAGA)

\section{Introduction}

Zadeh [1] proposed the classic fuzzy set to describe fuzzy problems with the membership degree in the closed interval [0,1]. Atanassov [2] presented the concept of the intuitionistic fuzzy set (IFS) to express fuzzy problems by the membership function and non-membership function. Smarandache [3] defined neutrosophic logic and introduced neutrosophic sets (NSs) to describe fuzzy problems by the truth, falsity, and indeterminacy membership functions. For easy engineering applications, some subclasses of NSs are defined. Wang et al. developed interval neutrosophic sets (INSs) [4] and single-valued neutrosophic sets (SVNSs) [5]. Ye presented simplified neutrosophic sets (SNSs) [6]. Wang et al. also presented multi-valued neutrosophic sets (MVNSs) [7]. Since then, INSs, SVNSs, SNSs and MVNSs have been widely applied in decision-making [8,9], medical diagnoses [10,11], and fault diagnoses [12,13]. Furthermore, many scholars developed some extension forms of NSs by combining neutrosophic sets with other sets, such as refined single-valued neutrosophic sets [14], intuitionistic neutrosophic soft sets [15], single-valued neutrosophic hesitant fuzzy sets [16], and rough neutrosophic sets [17].

Recently, Ali et al. [18,19] also put forward the concepts of neutrosophic cubic sets (NCSs) by combining neutrosophic sets with cubic sets, and defined internal and external NCSs. NCSs are described by two parts simultaneously, where the truth, falsity, and indeterminacy membership functions can be expressed by an interval value and an exact value simultaneously. Obviously, an NCS 
can be combined by an INS and an SVNS, and it contains much more information than an INS or an SVNS. Thus, some researchers have applied NCSs in decision-making problems effectively. Lu et al. [20] studied cosine measures of NCSs to deal with multiple attribute decision-making (MADM) problems. Banerjee et al. [21] established a grey relational analysis (GRA) method for MADM in NCS environment. Pramanik et al. [22] investigated a multi-criteria group decision making (MCGDM) method based on the similarity measure of NCSs. Moreover, aggregation operators have been widely applied in many MADM problems [23-29], and some aggregation operators have been studied for MADM problems in an NCS environment. Shi et al. [30] developed Dombi aggregation operators of NCSs for MADM. Zhan et al. [31] also proposed the neutrosophic cubic weighted arithmetic average (NCWAA) and neutrosophic cubic geometric weighted average (NCWGA) operators by extending the WAA and WGA operators to NCSs. However, the aforementioned NCWAA and NCWGA operators may cause some unreasonable results in some cases. In order to overcome the shortcomings of the NCWAA and NCWGA operators, this paper developed a new neutrosophic cubic hybrid weighted arithmetic and geometric aggregation (NCHWAGA) operator and analyzed its effectiveness for MADM by numerical examples. The main advantage of the proposed NCHWAGA operator can overcome the shortcomings of the existing NCWAA and NCWGA operators in some situations and obtain the moderate aggregation values.

The rest of the paper is organized as follows. Section 2 briefly introduces some basic concepts of NCSs and analyzes the shortcomings of the NCWAA and NCWGA operators. Then, Section 3 presents the NCHWAGA operator and investigated its properties. We establish a MADM approach based on the NCHWAGA operator in Section 4. Subsequently, Section 5 provides numerical examples with neutrosophic cubic information to demonstrate the application and effectiveness of the developed approach. Finally, Section 6 presents conclusions and possible future research.

\section{Preliminaries}

Some basic concepts and ranking methods of NCSs were introduced in this section.

Definition 1. [19,32] Let $\mathcal{Z}$ be a universal set. A NCS G in $\mathcal{Z}$ is denoted as follows:

$$
G=\left\{x,<T_{c}(x), V_{c}(x), F_{c}(x)>,<t_{c}(x), v_{c}(x), f_{c}(x)>\mid x \in \mathcal{Z}\right\},
$$

where $<T_{c}(x), V_{c}(x), F_{c}(x)>$ is an INS [4] in $\mathcal{Z}$, and the intervals $T_{c}(x)=\left[T_{c}{ }^{L}(x), T_{c}{ }^{U}(x)\right] \subseteq[0,1], V_{c}(x)=$ $\left[V_{c}{ }^{L}(x), V_{c}{ }^{U}(x)\right] \subseteq[0,1]$, and $F_{c}(x)=\left[F_{c}{ }^{L}(x), F_{c}{ }^{U}(x)\right] \subseteq[0,1]$ for $x \in \mathcal{Z}$ represent respectively the truth, indeterminacy, and falsity membership functions; then $\left\langle t_{c}(x), v_{c}(x), f_{c}(x)>\right.$ is an SVNS [3,5] in $\mathcal{Z}$, and $t_{c}(x)$, $v_{\mathcal{c}}(x), f_{c}(x) \in[0,1]$ for $x \in \mathcal{Z}$ represent the truth, indeterminacy, and falsity membership functions, respectively.

Then, we called a basic element $\left(x,<T_{c}(x), V_{c}(x), F_{c}(x)>,\left\langle t_{c}(x), v_{c}(x), f_{c}(x)>\right)\right.$ in an NCS $G$ a neutrosophic cubic number (NCN) [20]; for convenience, we denoted it as $g=(<$ $\left.\left[T^{\mathrm{L}}, T^{\mathrm{U}}\right],\left[V^{\mathrm{L}}, V^{\mathrm{U}}\right],\left[F^{\mathrm{L}}, F^{\mathrm{U}}\right]>,<t, v, f>\right)$, where $t, v, f \in[0,1]$ and $\left[T^{\mathrm{L}}, T^{\mathrm{U}}\right],\left[V^{\mathrm{L}}, V^{\mathrm{U}}\right],\left[F^{\mathrm{L}}, F^{\mathrm{U}}\right] \subseteq$ $[0,1]$ satisfy the condition $0 \leq T^{\mathrm{U}}+V^{\mathrm{U}}+F^{\mathrm{U}} \leq 3$ and $0 \leq t+v+f \leq 3$.

Then, an NCS $G=\left\{x,<T_{c}(x), V_{c}(x), F_{c}(x)>,<t_{c}(x), v_{c}(x), f_{c}(x)>\mid x \in \mathcal{Z}\right\}$ is called an internal NCS if $T_{c}{ }^{L}(x) \leq t_{c}(x) \leq T_{c}{ }^{U}(x), V_{c}{ }^{L}(x) \leq v_{c}(x) \leq V_{c}{ }^{U}(x)$, and $F_{c}{ }^{L}(x) \leq f_{c}(x) \leq F_{c}{ }^{U}(x)$ for $x \in \mathcal{Z}$; and an NCS $G$ is called an external NCS if $t_{c}(x) \notin\left(T_{c}{ }^{L}(x), T_{c}{ }^{U}(x)\right), v_{c}(x) \notin\left(V_{c}{ }^{L}(x), V_{c}^{U}(x)\right)$, and $f_{c}(x)$ $\notin\left(F_{c}{ }^{L}(x), F_{c}{ }^{U}(x)\right)$ for $x \in \mathcal{Z}[18,19]$.

Let $g_{1}=\left(<\left[T_{1} \mathrm{~L}, T_{1}^{\mathrm{U}}\right],\left[V_{1}^{\mathrm{L}}, V_{1}^{\mathrm{U}}\right],\left[F_{1} \mathrm{~L}, F_{1}^{\mathrm{U}}\right]>,<t_{1}, v_{1}, f_{1}>\right)$ and $g_{2}=\left(<\left[T_{2}{ }^{\mathrm{L}}\right.\right.$, $\left.\left.T_{2} \mathrm{U}\right],\left[V_{2} \mathrm{~L}, V_{2} \mathrm{U}\right],\left[F_{2}{ }^{\mathrm{L}}, F_{2} \mathrm{U}\right]>,<t_{2}, v_{2}, f_{2}>\right)$ be two NCNs, then there are following operational laws:

(1) $\left(g_{1}\right)^{\mathrm{C}}=\left(<\left[F_{1}^{\mathrm{L}}, F_{1}^{\mathrm{U}}\right],\left[1-V_{1}^{\mathrm{U}}, 1-V_{1}^{\mathrm{L}}\right],\left[T_{1}^{\mathrm{L}}, \mathrm{T}_{1}^{\mathrm{U}}\right]>,<f_{1}, 1-v_{1}, t_{1}>\right)$ (complement of $g_{1}$ );

$$
\begin{aligned}
g_{1} \oplus g_{2}= & \left(<\left[T_{1}^{\mathrm{L}}+T_{2}{ }^{\mathrm{L}}-T_{1}{ }^{\mathrm{L}} T_{2}{ }^{\mathrm{L}}, T_{1} \mathrm{U}+T_{2}{ }^{\mathrm{U}}-T_{1}{ }^{\mathrm{U}} T_{2} \mathrm{U}\right],\left[V_{1}{ }^{\mathrm{L}} V_{2}{ }^{\mathrm{L}}, V_{1}{ }^{\mathrm{U}} V_{2} \mathrm{U}\right],\right. \\
& {\left.\left[F_{1}{ }^{\mathrm{L}} F_{1}{ }^{\mathrm{L}}, F_{1} \mathrm{U} F_{2} \mathrm{U}\right]>,<t_{1}+t_{2}-t_{1} t_{2}, v_{1} v_{2}, f_{1} f_{2}>\right) ; }
\end{aligned}
$$




$$
\begin{aligned}
& g_{1} \otimes g_{2}=\left(<\left[T_{1}{ }^{\mathrm{L}} T_{2}{ }^{\mathrm{L}}, T_{1}{ }^{\mathrm{U}} T_{2} \mathrm{U}\right],\left[V_{1}^{\mathrm{L}}+V_{2}{ }^{\mathrm{L}}-V_{1}{ }^{\mathrm{L}} V_{2}{ }^{\mathrm{L}}, V_{1}^{\mathrm{U}}+V_{2}^{\mathrm{U}}-V_{1}{ }^{\mathrm{U}} V_{2}{ }^{\mathrm{U}}\right],\right. \\
& \left.\left[F_{1}^{\mathrm{L}}+F_{1}^{\mathrm{L}}-F_{1}^{\mathrm{L}} F_{1}^{\mathrm{L}}, F_{1}^{\mathrm{U}}+F_{2}^{\mathrm{U}}-F_{1}^{\mathrm{U}} F_{2}^{\mathrm{U}}\right]>,<t_{1} t_{2}, v_{1}+v_{2}-v_{1} v_{2}, f_{1}+f_{2}-f_{1} f_{2}>\right) ; \\
& \lambda g_{1}=\left(<\left[1-\left(1-T_{1}^{\mathrm{L}}\right)^{\lambda}, 1-\left(1-T_{1}^{\mathrm{U}}\right)^{\lambda}\right],\left[\left(V_{1}^{\mathrm{L}}\right)^{\lambda},\left(V_{1}^{\mathrm{U}}\right)^{\lambda}\right],\left[\left(F_{1}^{\mathrm{L}}\right)^{\lambda},\left(F_{1}^{\mathrm{U}}\right)^{\lambda}\right]>\right.\text {, } \\
& \left.<1-\left(1-t_{1}\right)^{\lambda},\left(v_{1}\right)^{\lambda},\left(f_{1}\right)^{\lambda}>\right) \text { for } \lambda>0 \text {; } \\
& \left(g_{1}\right)^{\lambda}=\left(<\left[\left(T_{1}{ }^{\mathrm{L}}\right)^{\lambda},\left(T_{1}^{\mathrm{U}}\right)^{\lambda}\right],\left[1-\left(1-V_{1}^{\mathrm{L}}\right)^{\lambda}, 1-\left(1-V_{1}{ }^{\mathrm{U}}\right)^{\lambda}\right], 1-\left(1-{F_{1}}^{\mathrm{L}}\right)^{\lambda},\right. \\
& \left.\left.1-\left(1-F_{1} \mathrm{U}\right)^{\lambda}\right]>,<\left(t_{1}\right)^{\lambda}, 1-\left(1-v_{1}\right)^{\lambda}, 1-\left(1-f_{1}\right)^{\lambda}>\right) \text { for } \lambda>0 \text {; }
\end{aligned}
$$

For any $\mathrm{NCN} g=\left(<\left[T^{\mathrm{L}}, T^{\mathrm{U}}\right],\left[V^{\mathrm{L}}, V^{\mathrm{U}}\right],\left[F^{\mathrm{L}}, F^{\mathrm{U}}\right]>,<t, v, f>\right)$, its score and accuracy functions [33] can be defined as follows:

$$
\begin{gathered}
\Psi(x)=\left[\left(4+T^{\mathrm{L}}+T^{\mathrm{U}}-V^{\mathrm{L}}-V^{\mathrm{U}}-F^{\mathrm{L}}-F^{\mathrm{U}}\right)+(2+t-v-f)\right] / 9 \\
\Gamma(x)=\left[\left(T^{\mathrm{L}}+T^{\mathrm{U}}-F^{\mathrm{L}}-F^{U}\right)+(t-f)\right] / 3
\end{gathered}
$$

Based on the functions $\Psi(x)$ and $\Gamma(x)$, two NCNs can be compared and ranked by definition as follows:

Definition 2. [33] Let $g_{1}=\left(<\left[T_{1}^{L}, T_{1}^{U}\right],\left[V_{1}^{L}, V_{1}^{U}\right],\left[F_{1}{ }^{L}, F_{1} U\right]>,<t_{1}, v_{1}, f_{1}>\right)$ and $g_{2}=$ $\left.\left[T_{2}{ }^{L}, T_{2}{ }^{U}\right],\left[V_{2}{ }^{L}, V_{2}{ }^{U}\right],\left[F_{2}{ }^{L}, F_{2}{ }^{U}\right]>,<t_{2}, v_{2}, f_{2}>\right)$ be two NCNs, then their comparative relations are defined as follows:

(i) If $\Psi\left(g_{1}\right)>\Psi\left(g_{2}\right)$, then $g_{1} \succ g_{2}$;

(ii) If $\Psi\left(g_{1}\right)=\Psi\left(g_{2}\right)$ and $\Gamma\left(g_{1}\right)>\Gamma\left(g_{2}\right)$, then $g_{1} \succ g_{2}$;

(iii) If $\Psi\left(g_{1}\right)=\Psi\left(g_{2}\right)$ and $\Gamma\left(g_{1}\right)=\Gamma\left(g_{2}\right)$, then $g_{1} \sim g_{2}$.

Assume that $g_{i}=\left(<\left[T_{i}^{\mathrm{L}}, T_{i}^{\mathrm{U}}\right],\left[V_{i}^{\mathrm{L}}, V_{i}^{\mathrm{U}}\right],\left[F_{i}^{\mathrm{L}}, F_{i}^{\mathrm{U}}\right]>,<t_{i}, v_{i}, f_{i}>\right)(i=1,2, \ldots, n)$ be a collection of NCNs. Then the NCWAA and NCWGA are provided, respectively, as follows [31]:

NCWAA $\left(g_{1}, g_{2}, \ldots, g_{n}\right)$

$$
=\sum_{i=1}^{n} \xi_{i} g_{i}=\left(\begin{array}{l}
\left\langle 1-\prod_{i=1}^{n}\left(1-T_{i}^{\mathrm{L}}\right)^{\xi_{i}}, 1-\prod_{i=1}^{n}\left(1-T_{i}^{\mathrm{U}}\right)^{\xi_{i}}\right],\left[\prod_{i=1}^{n}\left(V_{i}^{\mathrm{L}}\right)^{\xi_{i}}, \prod_{i=1}^{n}\left(V_{i}^{\mathrm{U}}\right)^{\xi_{i}}\right], \\
\left\langle\prod_{i=1}^{n}\left(F_{i}^{\mathrm{L}}\right)^{\xi_{i}}, \prod_{i=1}^{n}\left(F_{i}^{\mathrm{U}}\right)^{\xi_{i}}\right]
\end{array}\right\rangle,
$$

$\operatorname{NCWGA}\left(g_{1}, g_{2}, \ldots, g_{n}\right)$

$$
=\prod_{i=1}^{n} g_{i}^{\xi_{i}}=\left(\begin{array}{l}
\left\langle\prod_{i=1}^{n}\left(T_{i}^{\mathrm{L}}\right)^{\xi_{i}}, \prod_{i=1}^{n}\left(T_{i}^{\mathrm{U}}\right)^{\xi_{i}}\right],\left[1-\prod_{i=1}^{n}\left(1-V_{i}^{\mathrm{L}}\right)^{\xi_{i}}, 1-\prod_{i=1}^{n}\left(1-V_{i}^{\mathrm{U}}\right)^{\xi_{i}}\right], \\
{\left[1-\prod_{i=1}^{n}\left(1-F_{i}^{\mathrm{L}}\right)^{\xi_{i}}, 1-\prod_{i=1}^{n}\left(1-F_{i}^{\mathrm{U}}\right)^{\xi_{i}}\right]}
\end{array}\right\rangle,
$$

where $\zeta_{i} \in(i=1,2, \ldots, n)$, satisfying $\sum_{i=1}^{n} \xi_{i}=1$.

Although the above-weighted average and geometric operators were used for multi-criteria decision making [31], some unreasonable results are implied in the following two cases.

Case 1. Let $\left.\left.g_{1}=(<[0.001,0.002],[0,0],[0,0]\rangle,<0.001,0,0\right\rangle\right)$ and $\left.g_{2}=(<[0,1],[0,0],[0,0]\rangle,<1,0,0>\right)$ be two NCNs, with their weights $\zeta_{1}=0.9$ and $\zeta_{2}=0.1$, respectively.

Then, by Equations (3) and (4), NCWAA $\left(g_{1}, g_{2}\right)=(<[0.001,1],[0,0],[0,0]>,<1,0,0>)$ and NCWGA $\left(g_{1}, g_{2}\right)=(<[0,0.004],[0,0],[0,0]>,<0.002,0,0>)$. 
Case 2. Also take two NCNs $\left.\left.g_{1}=(<[0.001,0.002],[0,0],[0,0]\rangle,<0.001,0,0\right\rangle\right)$ and $g_{2}=(<[0,1],[0,0]$, $[0,0]>,\left\langle 1,0,0>\right.$ ) with their weights $\zeta_{1}=0.1$ and $\zeta_{2}=0.9$, respectively.

Then, by Equation (3) and (4), NCWAA $\left(g_{1}, g_{2}\right)=(<[0,1],[0,0],[0,0]>,<1,0,0>)$ and NCWGA $\left(g_{1}, g_{2}\right)=(<[0,0.537],[0,0],[0,0]>,<0.501,0,0>)$.

The above aggregated results indicate that the aggregated values of NCWAA $\left(g_{1}, g_{2}\right)$ operator tend to the maximum value, while the aggregated results of NCWGA $\left(g_{1}, g_{2}\right)$ operator tend to the maximum weight value. It is obvious that the NCWAA and NCWGA operators may cause unreasonable results of NCNs in some cases. In order to overcome the drawbacks, it is necessary to improve the NCWAA and NCWGA operators provided in [31]. Hence, in the next section, a new NCHWAGA is proposed by extending the hybrid arithmetic and geometric aggregation operators presented in $[34,35]$.

\section{Hybrid Arithmetic and Geometric Aggregation Operators of NCNs}

In this section, we present the NCHWAGA operator and investigated its properties.

\subsection{NCHWAGA Operator}

Definition 3. Let $g_{i}=\left(<\left[T_{i}^{L}, T_{i}^{U}\right],\left[V_{i}^{L}, V_{i}^{U}\right],\left[F_{i}^{L}, F_{i}^{U}\right]>,<t_{i}, v_{i}, f_{i}>\right)(i=1,2, \ldots, n)$ be a collection of NCNs. Then, the NCHWAGA operator is defined by:

$$
\operatorname{NCHWAGA}\left(g_{1}, g_{2}, \ldots, g_{n}\right)=\left(\sum_{i=1}^{n} \xi_{i} g_{i}\right)^{\rho}\left(\prod_{i=1}^{n} g_{i}^{\xi_{i}}\right)^{(1-\rho)}
$$

where $\rho \in[0,1]$; and $\zeta_{i}(i=1,2, \ldots, n)$ is the weight of $g_{i}(i=1,2, \ldots, n)$, satisfying $\zeta_{i} \in[0,1]$ and $\sum_{i=1}^{n} \xi_{i}=1$.

Theorem 1. Let $g_{i}=\left(<\left[T_{i}^{L}, T_{i}^{U}\right],\left[V_{i}^{L}, V_{i}^{U}\right],\left[F_{i}^{L}, F_{i}^{U}\right]>,<t_{i}, v_{i}, f_{i}>\right)(i=1,2, \ldots, n)$ be a collection of NCNs, and $\zeta_{i}(i=1,2, \ldots, n)$ be the corresponding weight of $g_{i}(i=1,2, \ldots, n)$, satisfying $\zeta_{i} \in[0,1]$ and $\sum_{i=1}^{n} \xi_{i}=1$. Then, the aggregated value of the NCHWAGA operator is also an NCN, which can be calculated by:

$$
\begin{aligned}
& \operatorname{NCHWAGA}\left(g_{1}, g_{2}, \ldots, g_{n}\right)=\left(\sum_{i=1}^{n} \xi_{i} g_{i}\right)^{\rho}\left(\prod_{i=1}^{n} g_{i}^{\xi_{i}}\right)^{(1-\rho)}
\end{aligned}
$$

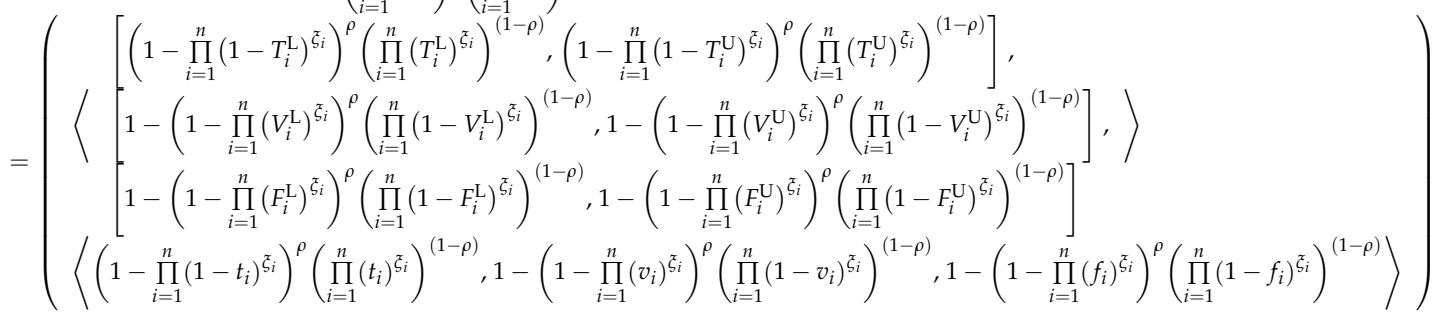

Proof. Based on the operational laws of NCNs in Section 2 and the NCWAA and NCWGA, we can obtain the following result:

$$
\begin{aligned}
& \text { NCHWAGA }\left(g_{1}, g_{2}, \ldots, g_{n}\right)=\left(\sum_{i=1}^{n} \xi_{i} g_{i}\right)^{\rho}\left(\prod_{i=1}^{n} g_{i}^{\tilde{z}_{i}}\right)^{(1-\rho)}
\end{aligned}
$$

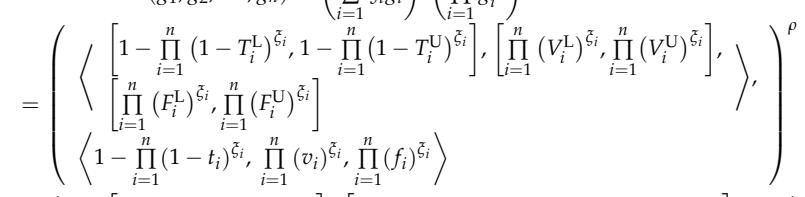

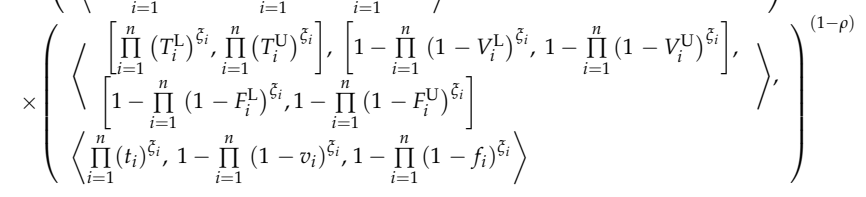




$$
\begin{aligned}
& =\left(\begin{array}{c}
{\left[\left(1-\prod_{i=1}^{n}\left(1-T_{i}^{\mathrm{L}}\right)^{\xi_{i}}\right)^{\rho},\left(1-\prod_{i=1}^{n}\left(1-T_{i}^{\mathrm{U}}\right)^{\xi_{i}}\right)^{\rho}\right]} \\
\left\langle\left[1-\left(1-\prod_{i=1}^{n}\left(V_{i}^{\mathrm{L}}\right)^{\xi_{i}}\right)^{\rho}, 1-\left(1-\prod_{i=1}^{n}\left(V_{i}^{\mathrm{U}}\right)^{\xi_{i}}\right)^{\rho}\right],\right. \\
{\left[1-\left(1-\prod_{i=1}^{n}\left(F_{i}^{\mathrm{L}}\right)^{\xi_{i}}\right)^{\rho}, 1-\left(1-\prod_{i=1}^{n}\left(F_{i}^{\mathrm{U}}\right)^{\xi_{i}}\right)^{\rho}\right]} \\
\left\langle\left(1-\prod_{i=1}^{n}\left(1-t_{i}\right)^{\xi_{i}}\right)^{\rho}, 1-\left(1-\prod_{i=1}^{n}\left(v_{i}\right)^{\xi_{i}}\right)^{\rho}, 1-\left(1-\prod_{i=1}^{n}\left(f_{i}\right)^{\xi_{i}}\right)^{\rho}\right\rangle
\end{array}\right) \\
& \times\left(\begin{array}{l}
{\left[\left(\prod_{i=1}^{n}\left(T_{i}^{\mathrm{L}}\right)^{\xi_{i}}\right)^{(1-\rho)},\left(\prod_{i=1}^{n}\left(T_{i}^{\mathrm{U}}\right)^{\xi_{i}}\right)^{(1-\rho)}\right],} \\
\left.\left\langle 1-\left(\prod_{i=1}^{n}\left(1-V_{i}^{\mathrm{L}}\right)^{\xi_{i}}\right)^{(1-\rho)}, 1-\left(\prod_{i=1}^{n}\left(1-V_{i}^{\mathrm{U}}\right)^{\xi_{i}}\right)^{(1-\rho)}\right],\right\rangle \\
{\left[1-\left(\prod_{i=1}^{n}\left(1-F_{i}^{\mathrm{L}}\right)^{\xi_{i}}\right)^{(1-\rho)}, 1-\left(\prod_{i=1}^{n}\left(1-F_{i}^{\mathrm{U}}\right)^{\xi_{i}}\right)^{(1-\rho)}\right]} \\
\left\langle\left(\prod_{i=1}^{n}\left(t_{i}\right)^{\xi_{i}}\right)^{(1-\rho)}, 1-\left(\prod_{i=1}^{n}\left(1-v_{i}\right)^{\xi_{i}}\right)^{(1-\rho)}, 1-\left(\prod_{i=1}^{n}\left(1-f_{i}\right)^{\tilde{\xi}_{i}}\right)^{(1-\rho)}\right\rangle
\end{array}\right)
\end{aligned}
$$

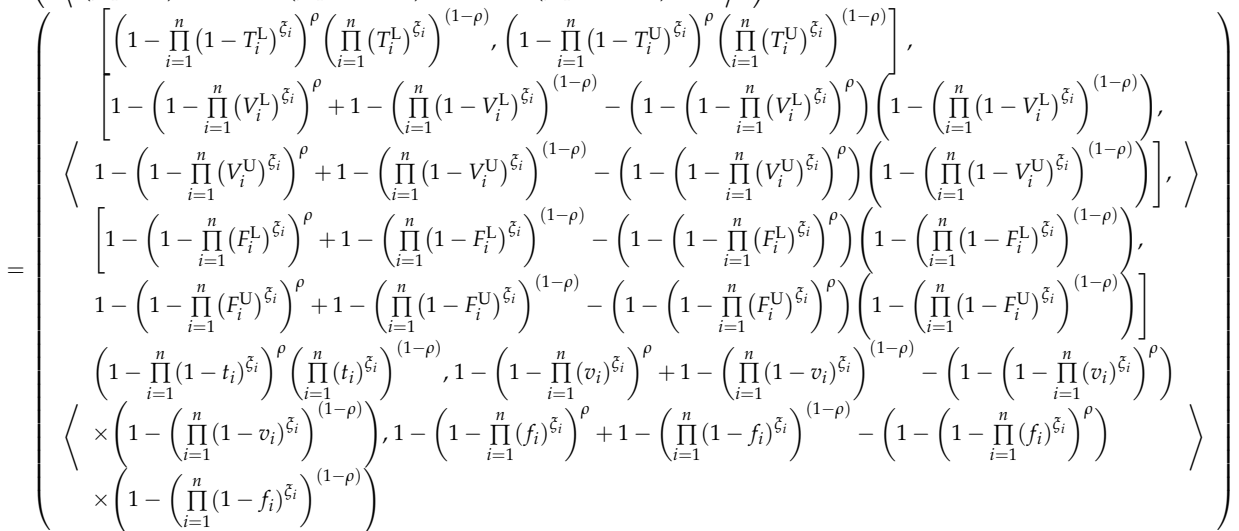

$$
\begin{aligned}
& \left(\begin{array}{l}
\quad\left[\left(1-\prod_{i=1}^{n}\left(1-T_{i}^{\mathrm{L}}\right)^{\xi_{i}}\right)^{\rho}\left(\prod_{i=1}^{n}\left(T_{i}^{\mathrm{L}}\right)^{\xi_{i}}\right)^{(1-\rho)},\left(1-\prod_{i=1}^{n}\left(1-T_{i}^{\mathrm{U}}\right)^{\xi_{i}}\right)^{\rho}\left(\prod_{i=1}^{n}\left(T_{i}^{\mathrm{U}}\right)^{\xi_{i}}\right)^{(1-\rho)}\right] \\
{\left[\begin{array}{l}
1-\left(1-\prod_{i=1}^{n}\left(V_{i}^{\mathrm{L}}\right)^{\xi_{i}}\right)^{\rho}+1-\left(\prod_{i=1}^{n}\left(1-V_{i}^{\mathrm{L}}\right)^{\xi_{i}}\right)^{(1-\rho)}-\left(1-\left(\prod_{i=1}^{n}\left(1-V_{i}^{\mathrm{L}}\right)^{\xi_{i}}\right)^{(1-\rho)}\right) \\
+\left(1-\prod_{i=1}^{n}\left(V_{i}^{\mathrm{L}}\right)^{\xi_{i}}\right)^{\rho}-\left(1-\prod_{i=1}^{n}\left(V_{i}^{\mathrm{L}}\right)^{\xi_{i}}\right)^{\rho}\left(\prod_{i=1}^{n}\left(1-V_{i}^{\mathrm{L}}\right)^{\xi_{i}}\right)^{(1-\rho)}
\end{array}\right),} \\
\left.\left(\begin{array}{l}
1-\left(1-\prod_{i=1}^{n}\left(V_{i}^{\mathrm{U}}\right)^{\xi_{i}}\right)^{\rho}+1-\left(\prod_{i=1}^{n}\left(1-V_{i}^{\mathrm{U}}\right)^{\xi_{i}}\right)^{(1-\rho)}-\left(1-\left(\prod_{i=1}^{n}\left(1-V_{i}^{\mathrm{U}}\right)^{\xi_{i}}\right)^{(1-\rho)}\right) \\
+\left(1-\prod_{i=1}^{n}\left(V_{i}^{\mathrm{U}}\right)^{\xi_{i}}\right)^{\rho}-\left(1-\prod_{i=1}^{n}\left(V_{i}^{\mathrm{U}}\right)^{\xi_{i}}\right)^{\rho}\left(\prod_{i=1}^{n}\left(1-V_{i}^{\mathrm{U}}\right)^{\xi_{i}}\right)^{(1-\rho)}
\end{array}\right),\right\rangle
\end{array}\right. \\
& =\left[\begin{array}{l}
1-\left(1-\prod_{i=1}^{n}\left(F_{i}^{\mathrm{L}}\right)^{\xi_{i}}\right)^{\rho}+1-\left(\prod_{i=1}^{n}\left(1-F_{i}^{\mathrm{L}}\right)^{\xi_{i}}\right)^{(1-\rho)}-\left(1-\left(\prod_{i=1}^{n}\left(1-F_{i}^{\mathrm{L}}\right)^{\xi_{i}}\right)^{(1-\rho)}\right) \\
+\left(1-\prod_{i=1}^{n}\left(F_{i}^{\mathrm{L}}\right)^{\xi_{i}}\right)^{\rho}-\left(1-\prod_{i=1}^{n}\left(F_{i}^{\mathrm{L}}\right)^{\xi_{i}}\right)^{\rho}\left(\prod_{i=1}^{n}\left(1-F_{i}^{\mathrm{L}}\right)^{\xi_{i}}\right)^{(1-\rho)}
\end{array}\right), \\
& \left.\left(\begin{array}{l}
1-\left(1-\prod_{i=1}^{n}\left(F_{i}^{\mathrm{U}}\right)^{\xi_{i}}\right)^{\rho}+1-\left(\prod_{i=1}^{n}\left(1-F_{i}^{\mathrm{U}}\right)^{\xi_{i}}\right)^{(1-\rho)}-\left(1-\left(\prod_{i=1}^{n}\left(1-F_{i}^{\mathrm{U}}\right)^{\xi_{i}}\right)^{(1-\rho)}\right) \\
+\left(1-\prod_{i=1}^{n}\left(F_{i}^{\mathrm{U}}\right)^{\xi_{i}}\right)^{\rho}-\left(1-\prod_{i=1}^{n}\left(F_{i}^{\mathrm{U}}\right)^{\xi_{i}}\right)^{\rho}\left(\prod_{i=1}^{n}\left(1-F_{i}^{\mathrm{U}}\right)^{\xi_{i}}\right)^{(1-\rho)}
\end{array}\right)\right] \\
& \left(1-\prod_{i=1}^{n}\left(1-t_{i}\right)^{\xi_{i}}\right)^{\rho}\left(\prod_{i=1}^{n}\left(t_{i}\right)^{\xi_{i}}\right)^{i=1}, 1-\left(1-\prod_{i=1}^{n}\left(v_{i}\right)^{\xi_{i}}\right)^{\rho}+1-\left(\prod_{i=1}^{n}\left(1-v_{i}\right)^{\xi_{i}}\right)^{(1-\rho)}
\end{aligned}
$$

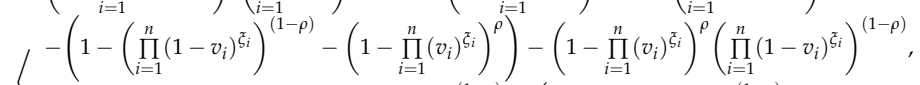

$$
\begin{aligned}
& \left.\begin{array}{l}
\left\langle 1-\left(1-\prod_{i=1}^{n}\left(f_{i}\right)^{\xi_{i}}\right)^{\rho}+1-\left(\prod_{i=1}^{n}\left(1-f_{i}\right)^{\xi_{i}}\right)^{(1-\rho)}-\left(1-\left(\prod_{i=1}^{n}\left(1-f_{i}\right)^{\xi_{i}}\right)^{(1-\rho)}-\left(1-\prod_{i=1}^{n}\left(f_{i}\right)^{\xi_{i}}\right)^{\rho}\right)\right\rangle \\
-\left(1-\prod_{i=1}^{n}\left(f_{i}\right)^{\xi_{i}}\right)^{\rho}\left(\prod_{i=1}^{n}\left(1-f_{i}\right)^{\xi_{i}}\right)^{(1-\rho)}
\end{array}\right) \\
& =\left(\begin{array}{l}
{\left[\begin{array}{l}
\left.\left(1-\prod_{i=1}^{n}\left(1-T_{i}^{\mathrm{L}}\right)^{\xi_{i}}\right)^{\rho}\left(\prod_{i=1}^{n}\left(T_{i}^{\mathrm{L}}\right)^{\xi_{i}}\right)^{(1-\rho)},\left(1-\prod_{i=1}^{n}\left(1-T_{i}^{\mathrm{U}}\right)^{\xi_{i}}\right)^{\rho}\left(\prod_{i=1}^{n}\left(T_{i}^{\mathrm{U}}\right)^{\xi_{i}}\right)^{(1-\rho)}\right], \\
\left.\left\langle 1-\left(1-\prod_{i=1}^{n}\left(V_{i}^{\mathrm{L}}\right)^{\xi_{i}}\right)^{\rho}\left(\prod_{i=1}^{n}\left(1-V_{i}^{\mathrm{L}}\right)^{\xi_{i}}\right)^{(1-\rho)}, 1-\left(1-\prod_{i=1}^{n}\left(V_{i}^{\mathrm{U}}\right)^{\xi_{i}}\right)^{\rho}\left(\prod_{i=1}^{n}\left(1-V_{i}^{\mathrm{U}}\right)^{\xi_{i}}\right)^{(1-\rho)}\right],\right\rangle \\
\left.1-\left(1-\prod_{i=1}^{n}\left(F_{i}^{\mathrm{L}}\right)^{\xi_{i}}\right)^{\rho}\left(\prod_{i=1}^{n}\left(1-F_{i}^{\mathrm{L}}\right)^{\xi_{i}}\right)^{(1-\rho)}, 1-\left(1-\prod_{i=1}^{n}\left(F_{i}^{\mathrm{U}}\right)^{\xi_{i}}\right)^{\rho}\left(\prod_{i=1}^{n}\left(1-F_{i}^{\mathrm{U}}\right)^{\xi_{i}}\right)^{(1-\rho)}\right]
\end{array}\right]} \\
\left\langle\left(1-\prod_{i=1}^{n}\left(1-t_{i}\right)^{\xi_{i}}\right)^{\rho}\left(\prod_{i=1}^{n}\left(t_{i}\right)^{\xi_{i}}\right)^{(1-\rho)}, 1-\left(1-\prod_{i=1}^{n}\left(v_{i}\right)^{\xi_{i}}\right)^{\rho}\left(\prod_{i=1}^{n}\left(1-v_{i}\right)^{\xi_{i}}\right)^{(1-\rho)}, 1-\left(1-\prod_{i=1}^{n}\left(f_{i}\right)^{\xi_{i}}\right)^{\rho}\left(\prod_{i=1}^{n}\left(1-f_{i}\right)^{\xi_{i}}\right)^{(1-\rho)}\right\rangle
\end{array}\right)
\end{aligned}
$$

The proof is finished. Hence, Theorem 1 is true.

Let $g_{i}=\left(<\left[T_{i}^{\mathrm{L}}, T_{i}^{\mathrm{U}}\right],\left[V_{i}^{\mathrm{L}}, V_{i}^{\mathrm{U}}\right],\left[F_{i}^{\mathrm{L}}, F_{i}^{\mathrm{U}}\right]>,<t_{i}, v_{i}, f_{i}>\right)(i=1,2, \ldots, n)$ be a collection of NCNs. Corresponding to the properties of the NCWAA and NCWGA operators [31], the NCNHWAGA operator also satisfies these properties: 
(i) Idempotency: If $g_{i}=g$ for $i=1,2, \ldots, n$, then NCHWAGA $\left(g_{1}, g_{2}, \ldots, g_{\mathrm{n}}\right)=g$.

(ii) Boundedness: If $g_{\min }=\min \left(g_{1}, g_{2}, \ldots, g_{n}\right)$ and $g_{\max }=\max \left(g_{1}, g_{2}, \ldots, g_{n}\right)$ for $i=1,2, \ldots, n$, then $g_{\min } \leq$ NCHWAGA $\left(g_{1}, g_{2}, \ldots, g_{\mathrm{n}}\right) \leq g_{\max }$.

(iii) Monotonicity: If $g_{\mathrm{i}} \leq g_{\mathrm{i}}^{*}$ for $i=1,2, \ldots, n$, then NCHWAGA $\left(g_{1}, g_{2}, \ldots, g_{\mathrm{n}}\right) \leq$ NCHWAGA $\left(g_{1}{ }^{*}, g_{2}{ }^{*}, \ldots, g_{\mathrm{n}}{ }^{*}\right)$.

For different values of $\rho \in[0,1]$, we can discuss the families of the NCHWAGA operator in some special cases as follows:

(i) The NCHWAGA operator reduces to the NCWAA operator [31] if $\rho=1$.

(ii) The NCHWAGA operator reduces to the NCWGA operator [31] if $\rho=0$.

(iii) The NCHWAGA operator is the mean of the NCWAA and NCWGA operators [31] if $\rho=0.5$.

\subsection{Numerical Example}

We still consider the above two numerical examples in Section 2 to demonstrate the effectiveness of the presented NCHWAGA operator. Generally taking $\rho=0.5$, we calculate aggregated values of the NCHWAGA operator.

For Case 1: Let two NCNs $\left.\left.g_{1}=(<[0.001,0.002],[0,0],[0,0]\rangle,<0.001,0,0\right\rangle\right)$ and $g_{2}=(<[0,1],[0,0]$, $[0,0]>,<1,0,0>)$ with their weights $\zeta_{1}=0.9$ and $\zeta_{2}=0.1$, by Equation (6), we obtain NCHWAGA $\left(g_{1}, g_{2}\right)=$ $(<[0,0.061],[0,0],[0,0]\rangle,<0.045,0,0\rangle)$, which is between NCWAA $\left(g_{1}, g_{2}\right)=(<[0.001,1],[0,0],[0,0]\rangle$, $<1,0,0>)$ and NCWGA $\left(g_{1}, g_{2}\right)=(<[0,0.004],[0,0],[0,0]>,<0.002,0,0>)$.

For Case 2: Also take two NCNs $\left.\left.g_{1}=(<[0.001,0.002],[0,0],[0,0]\rangle,<0.001,0,0\right\rangle\right)$ and $g_{2}=(<[0,1]$, $[0,0],[0,0]>,<1,0,0>)$ with their weights $\zeta_{1}=0.1$ and $\zeta_{2}=0.9$, then, by Equation (6), we get NCHWAGA $\left.\left(g_{1}, g_{2}\right)=(<[0,0.733],[0,0],[0,0]>,<0.708,0,0\rangle\right)$, which is between NCWAA $\left(g_{1}, g_{2}\right)=(<[0,1],[0,0]$, $[0,0]\rangle,\langle 1,0,0\rangle)$ and NCWGA $\left.\left(g_{1}, g_{2}\right)=(<[0,0.537],[0,0],[0,0]\rangle,\langle 0.501,0,0\rangle\right)$.

In the above two cases, we can obtain the moderate values by the NCHWAGA operator. Obviously, the NCHWAGA operator can overcome the drawbacks of the NCWAA and NCWGA provided in Reference [31].

\section{MADM Method Using the NCHWAGA Operator}

In this section, we provide a MADM method based on the NCHWAGA operator to deal with neutrosophic cubic information.

In a MADM problem, assume that $G=\left\{G_{1}, G_{2}, \ldots, G_{k}\right\}$ is a set of $k$ alternatives and $P=\left\{P_{1}, P_{2}\right.$, $\left.\ldots, P_{n}\right\}$ is a set of attributes. Suppose that the weight vector of $P$ is $\omega_{P}=\left\{\omega_{P_{1}}, \omega_{P_{2}}, \ldots, \omega_{P_{n}}\right\}$ with $\omega_{P_{j}} \in[0,1]$ and $\sum_{j=1}^{n} \omega_{P_{j}}=1$. The evaluation value of an alternative $G_{i}$ under an attribute $P_{j}$ can be expressed using an NCN $g_{i j}=\left(<\left[T_{i j}^{L}, T_{I j}^{U}\right],\left[V_{i j}^{L}, V_{i j}^{U}\right],\left[F_{i j}^{L}, F_{i j}^{U}\right]>,<t_{i j}, v_{i j}, f_{i j}>\right)(i=1,2, \ldots, k$; $j=1,2, \ldots, n)$, where $\left[T_{i j}^{L}, T_{i j}^{U}\right],\left[V_{i j}^{L}, V_{i j}^{U}\right],\left[F_{i j}^{L}, F_{i j}^{U}\right] \subseteq[0,1]$, and $t_{i j}, v_{i j}, f_{i j} \in[0,1]$. Then, we can construct a decision matrix $G=\left(g_{i j}\right)_{k \times n}$ with the NCN information, and provide the following MADM procedures based on the proposed NCHWAGA operator:

Step 1. Calculate the aggregated value of $g_{i}$ for each alternative $G_{i}(i=1,2, \ldots, k)$ using the NCHWAGA operator:

$$
\begin{aligned}
& g_{i}=\operatorname{NCHWAGA}\left(g_{i 1}, g_{i 2}, \ldots, g_{i n}\right)=\left(\sum_{j=1}^{n} \omega_{p_{j}} g_{i j}\right)^{\rho}\left(\prod_{j=1}^{n} g_{i j}^{\omega_{p_{j}}}\right)^{(1-\rho)} \\
& =\left(\begin{array}{c}
{\left[\left(1-\prod_{j=1}^{n}\left(1-T_{i j}^{\mathrm{L}}\right)^{\omega_{p_{j}}}\right)^{\rho}\left(\prod_{j=1}^{n}\left(T_{i j}^{\mathrm{L}}\right)^{\omega_{p_{j}}}\right)^{(1-\rho)},\left(1-\prod_{j=1}^{n}\left(1-T_{i}^{\mathrm{U}}\right)^{\omega_{p_{j}}}\right)^{\rho}\left(\prod_{j=1}^{n}\left(T_{i j}^{\mathrm{U}}\right)^{\omega_{p_{j}}}\right)^{(1-\rho)}\right],} \\
\left\langle\left[1-\left(1-\prod_{j=1}^{n}\left(V_{i j}^{\mathrm{L}}\right)^{\omega_{p_{j}}}\right)^{\rho}\left(\prod_{j=1}^{n}\left(1-V_{i j}^{\mathrm{L}}\right)^{\omega_{p_{j}}}\right)^{(1-\rho)}, 1-\left(1-\prod_{j=1}^{n}\left(V_{i j}^{\mathrm{U}}\right)^{\omega_{p_{j}}}\right)^{\rho}\left(\prod_{j=1}^{n}\left(1-V_{i j}^{\mathrm{U}}\right)^{\omega_{p_{j}}}\right)^{(1-\rho)}\right],\right\rangle \\
{\left[1-\left(1-\prod_{j=1}^{n}\left(F_{i j}^{\mathrm{L}}\right)^{\omega_{p_{j}}}\right)^{\rho}\left(\prod_{j=1}^{n}\left(1-F_{i j}^{\mathrm{L}}\right)^{\omega_{p_{j}}}\right)^{(1-\rho)}, 1-\left(1-\prod_{j=1}^{n}\left(F_{j}^{\mathrm{U}}\right)^{\omega_{p_{j}}}\right)^{\rho}\left(\prod_{j=1}^{n}\left(1-F_{i j}^{\mathrm{U}}\right)^{\omega_{p_{j}}}\right)^{(1-\rho)}\right]} \\
\left\langle\left(1-\prod_{j=1}^{n}\left(1-t_{i j}\right)^{\omega_{p_{j}}}\right)^{\rho}\left(\prod_{j=1}^{n}\left(t_{i j}\right)^{\omega_{p_{j}}}\right)^{(1-\rho)}, 1-\left(1-\prod_{j=1}^{n}\left(v_{i j}\right)^{\omega_{p_{j}}}\right)^{\rho}\left(\prod_{j=1}^{n}\left(1-v_{i j}\right)^{\omega_{p_{j}}}\right)^{(1-\rho)}, 1-\left(1-\prod_{j=1}^{n}\left(f_{i j}\right)^{\omega_{p_{j}}}\right)^{\rho}\left(\prod_{j=1}^{n}\left(1-f_{i j}\right)^{\omega_{p_{j}}}\right)^{(1-\rho)}\right\rangle
\end{array}\right)
\end{aligned}
$$


where $\omega_{P_{j}} \in[0,1]$ and $\sum_{j=1}^{n} \omega_{P_{j}}=1$ for $j=1,2, \ldots, n$.

Step 2. Obtain the score values of $\Psi(x)$ (the accuracy degrees of $\Gamma(x)$ if necessary) of the collective NCN $g_{i}(i=1,2, \ldots, k)$ by Equations (1) and (2).

Step 3. Rank all the alternatives corresponding to the values of $\Psi(x)$ and $\Gamma(x)$, and select the best one(s) based on the largest value.

Step 4. End.

\section{Illustrative Example and Comparison Analysis}

This section introduces an illustrative example adapted from Reference [20] to demonstrate the application of the above MADM method. A company wants to invest some money in one of the four possible alternatives $G_{i}(i=1,2,3,4) . G_{1}, G_{2}, G_{3}$ and $G_{4}$ represent a textile company, an automobile company, a computer company, and a software company, respectively. The four alternatives need to be evaluated according to the three attributes $P_{j}(j=1,2,3) . P_{1}, P_{2}$ and $P_{3}$ represent respectively the risk, the growth, and the environmental impact. Corresponding to the three attributes, the weight vector is $\omega_{P}=(0.32,0.38,0.3)$. When the decision maker evaluates the four alternatives $G_{i}(i=1,2,3,4)$ based on the three attributes $P_{j}(j=1,2,3)$ with the NCN information, the decision matrix can be established as shown in Table 1.

Table 1. The decision matrix with the neutrosophic cubic number (NCN) information.

\begin{tabular}{cccc}
\hline Alternative & Attribute $\left(\boldsymbol{P}_{\mathbf{1}}\right)$ & Attribute $\left(\boldsymbol{P}_{\mathbf{2}}\right)$ & Attribute $\left(\boldsymbol{P}_{\mathbf{3}}\right)$ \\
\hline \multirow{2}{*}{$G_{1}$} & $(<[0.5,0.6],[0.1,0.3]$, & $(<[0.5,0.6],[0.1,0.3]$, & $(<[0.6,0.8],[0.2,0.3]$, \\
& $[0.2,0.4]>,<0.2,0.6,0.3>)$ & $[0.2,0.4]>,<0.2,0.6,0.3>)$ & $[0.1,0.2]>,<0.7,0.2,0.1>)$ \\
$G_{2}$ & $(<[0.6,0.8],[0.1,0.2]$, & $(<[0.6,0.7],[0.1,0.2]$, & $(<[0.6,0.7],[0.3,0.4]$, \\
& $[0.2,0.3]>,<0.7,0.1,0.2>)$ & $[0.2,0.3]>,<0.6,0.3,0.4>)$ & $[0.1,0.2]>,<0.7,0.4,0.2>)$ \\
$G_{3}$ & $(<[0.4,0.6],[0.2,0.3]$, & $(<[0.5,0.6],[0.2,0.3]$, & $(<[0.5,0.7],[0.2,0.3]$, \\
& $[0.1,0.3]>,<0.6,0.2,0.2>)$ & $[0.3,0.4]>,<0.6,0.3,0.4>)$ & $[0.3,0.4]>,<0.6,0.2,0.3>)$ \\
$G_{4}$ & $(<[0.7,0.8],[0.1,0.2]$, & $(<[0.6,0.7],[0.1,0.2]$, & $(<[0.6,0.7],[0.3,0.4]$, \\
& $[0.1,0.2]>,<0.8,0.1,0.2>)$ & $[0.1,0.3]>,<0.7,0.1,0.2>)$ & $[0.2,0.3]>,<0.7,0.3,0.2>)$ \\
\hline
\end{tabular}

Then, we apply the NCHWAGA operator to handle the MADM problem as follows:

Step 1. By Equation (7) for $\rho=0.5$, we calculate the aggregated value of the collective $\mathrm{NCN} g_{i}$ for the each alternative $G_{i}(i=1,2,3,4)$ as follows:

\begin{tabular}{c}
\hline$g_{1}=(<[0.5302,0.6645],[0.1272,0.3000],[0.1669,0.3355]>,<0.3430,0.4709,0.2306>)$ \\
\hline$g_{2}=(<[0.6000,0.7335],[0.1523,0.2563],[0.1669,0.2685]>,<0.6628,0.2525,0.2346>)$ \\
\hline$g_{3}=(<[0.4677,0.6307],[0.2000,0.3000],[0.2264,0.3672]>,<0.6000,0.2365,0.3025>)$ \\
\hline$g_{4}=(<[0.6328,0.7335],[0.1523,0.2563],[0.1272,0.2665]>,<0.7335,0.1523,0.2000>)$ \\
\hline
\end{tabular}

Step 2. By Equation (1), we calculate the score values of $\Psi\left(g_{i}\right)$ for the alternatives $G_{i}(i=1,2,3,4)$ as the follows:

$$
\Psi\left(g_{1}\right)=0.6563, \Psi\left(g_{2}\right)=0.7405, \Psi\left(g_{3}\right)=0.6740, \Psi\left(g_{4}\right)=0.7717 .
$$

Step 3. According to $\Psi\left(g_{4}\right)>\Psi\left(g_{2}\right)>\Psi\left(g_{3}\right)>\Psi\left(g_{1}\right)$, the ranking of the alternatives is $G_{4} \succ G_{2} \succ G_{3} \succ$ $G_{1}$. So, the alternative $G_{4}$ is the best one.

Compared with the MADM method introduced in Reference [20], Table 2 lists the decision results based on the NCHWAGA operator and cosine similarity measures of the NCSs. Obviously, the best alternatives and the ranking orders based on the NCHWAGA operator proposed in this paper are the same as in Reference [20].

For further relative comparison, Table 3 lists the MADM results using the NCHWAGA operator proposed in this paper and the NCWAA and NCWGA operators provided in Reference [31], respectively. The results listed in Table 3 show that the aggregated values of the NCHWAGA operator 
tend to the moderate values between the aggregated results of the NCWAA and NCWGA operators. Then, the ranking orders based on the NCHWAGA operator have little difference with the NCWAA and NCWGA operators. However, the best alternative given in all the MADM methods is identical. Furthermore, the results in Table 3 also show that the aggregated values of the NCHWAGA operator tend to moderate values between the aggregated values of the NCWAA and NCWGA operators in [31]. Therefore, the NCHWAGA operator can overcome the drawbacks of the NCWAA and NCWGA operators, and it is more effective and more suitable than the NCWAA and NCWGA operators to handle MADM problems under a neutrosophic cubic environment in some cases.

Table 2. Decision results based on the neutrosophic cubic hybrid weighted arithmetic and geometric aggregation (NCHWAGA) operator and cosine similarity measures.

\begin{tabular}{cccc}
\hline MADM Method & $\begin{array}{c}\text { Score Values } \\
\text { (Cosine Measures Value) }\end{array}$ & Ranking Order & $\begin{array}{c}\text { The Best } \\
\text { Alternative }\end{array}$ \\
\hline NCHWAGA $(\rho=0.5)$ & $0.6563,0.7405,0.6740,0.7717$ & $G_{4} \succ G_{2} \succ G_{1} \succ G_{3}$ & $G_{4}$ \\
Cosine Measure $S_{w 1}[20]$ & $0.9564,0.9855,0.9596,0.9945$ & $G_{4} \succ G_{2} \succ G_{1} \succ G_{3}$ & $G_{4}$ \\
Cosine Measure $S_{w 2}[20]$ & $0.9769,0.9944,0.9795,0.9972$ & $G_{4} \succ G_{2} \succ G_{1} \succ G_{3}$ & $G_{4}$ \\
Cosine Measure $S_{w 3}[20]$ & $0.9892,0.9959,0.9897,0.9989$ & $G_{4} \succ G_{2} \succ G_{1} \succ G_{3}$ & $G_{4}$ \\
\hline
\end{tabular}

Table 3. Decision results based on different aggregation operators.

\begin{tabular}{|c|c|c|c|c|}
\hline $\begin{array}{l}\text { Aggregation } \\
\text { Operator }\end{array}$ & Aggregated Result & Score Value & Ranking Order & $\begin{array}{l}\text { The Best } \\
\text { Alternative }\end{array}$ \\
\hline \multirow{4}{*}{$\begin{array}{l}\text { NCHWAGA } \\
\quad(\rho=0.5)\end{array}$} & $\begin{array}{l}g_{1}=(<[0.5302,0.6645],[0.1272,0.3000] \\
[0.1669,0.3355]>,<0.3430,0.4709,0.2306>)\end{array}$ & $\Psi\left(g_{1}\right)=0.6563$ & \multirow{4}{*}{$G_{4} \succ G_{2} \succ G_{1} \succ G_{3}$} & \multirow{4}{*}{$G_{4}$} \\
\hline & $\begin{array}{l}g_{2}=(<[0.6000,0.7335],[0.1523,0.2563] \\
[0.1669,0.2685]>,<0.6628,0.2525,0.2346>)\end{array}$ & $\Psi\left(g_{2}\right)=0.7405$ & & \\
\hline & $\begin{array}{l}g_{3}=(<[0.4677,0.6307],[0.2000,0.3000] \\
[0.2264,0.3672]>,<0.6000,0.2365,0.3025>)\end{array}$ & $\Psi\left(g_{3}\right)=0.6740$ & & \\
\hline & $\begin{array}{l}g_{4}=(<[0.6328,0.7335],[0.1523,0.2563] \\
[0.1272,0.2665]>,<0.7335,0.1523,0.2000>)\end{array}$ & $\Psi\left(g_{4}\right)=0.7717$ & & \\
\hline \multirow{4}{*}{ NCWAA [31] } & $\begin{array}{l}g_{1}=(<[0.5324,0.6751],[0.1231,0.3000] \\
[0.1625,0.3249]>,<0.4039,0.4315,0.2158>)\end{array}$ & $\Psi\left(g_{1}\right)=0.6726$ & \multirow{4}{*}{$G_{4} \succ G_{2} \succ G_{3} \succ G_{1}$} & \multirow{4}{*}{$G_{4}$} \\
\hline & $\begin{array}{l}g_{2}=(<[0.6000,0.7365],[0.1390,0.2462] \\
[0.1625,0.2656]>,<0.6653,0.2301,0.2114>)\end{array}$ & $\Psi\left(g_{2}\right)=0.7497$ & & \\
\hline & $\begin{array}{l}g_{3}=(<[0.4700,0.6331],[0.2000,0.3000] \\
[0.2111,0.3648]>,<0.6000,0.2333,0.2939>)\end{array}$ & $\Psi\left(g_{3}\right)=0.6778$ & & \\
\hline & $\begin{array}{l}g_{4}=(<[0.6352,0.7365],[0.1390,0.2462] \\
[0.1231,0.2635]>,<0.7365,0.1390,0.2000>)\end{array}$ & $\Psi\left(g_{4}\right)=0.7775$ & & \\
\hline \multirow{4}{*}{ NCWGA [31] } & $\begin{array}{l}g_{1}=(<[0.5281,0.6541],[0.1312,0.3000] \\
[0.1712,0.3459]>,<0.2912,0.5075,0.2452>)\end{array}$ & $\Psi\left(g_{1}\right)=0.6414$ & \multirow{4}{*}{$G_{4} \succ G_{2} \succ G_{3} \succ G_{1}$} & \multirow{4}{*}{$G_{4}$} \\
\hline & $\begin{array}{l}g_{2}=(<[0.6000,0.7306],[0.1654,0.2661] \\
[0.1712,0.2714]>,<0.6602,0.2757,0.2571>)\end{array}$ & $\Psi\left(g_{2}\right)=0.7315$ & & \\
\hline & $\begin{array}{l}g_{3}=(<[0.4655,0.6284],[0.2000,0.3000] \\
[0.2414,0.3697]>,<0.6000,0.2396,0.3110>)\end{array}$ & $\Psi\left(g_{3}\right)=0.6703$ & & \\
\hline & $\begin{array}{l}g_{4}=(<[0.6303,0.7306],[0.1654,0.2661] \\
[0.1312,0.2694]>,<0.7306,0.1654,0.2000>)\end{array}$ & $\Psi\left(g_{4}\right)=0.7660$ & & \\
\hline
\end{tabular}

\section{Conclusions}

This paper developed the NCHWAGA operator of NCSs and investigated its properties. The main advantage of the proposed NCHWAGA operator can overcome the drawbacks implied by the existing NCWAA and NCWGA operators [31] in some cases and reach the moderate aggregated values. Then, the MADM method based on the NCHWAGA operator was established under an NCS environment. Finally, we provided an illustrative example to demonstrate the application of the established MADM method. By comparison, we found that the developed MADM method was more effective and more suitable to solve decision-making problems with neutrosophic cubic information in some cases. In the real world, a refined neutrosophic set [14] is very suitable to express complex problems of decision-making, since it can be described by its refined types of sub-truths, 
sub-indeterminacies, and sub-falsities. Therefore, we shall further extend the NCHWAGA operator to neutrosophic refined cubic sets for MADM by using the refined neutrosophic sets. In addition, the proposed method will be also extended to neutrosophic cubic oversets/undersets/offsets using the neutrosophic overset/underset/offset [36] in the future.

Author Contributions: L.S. investigated the NCHWAGA operator and its MADM method; Y.Y. analyzed the numerical examples; we wrote this paper together.

Funding: This research received no external funding.

Acknowledgments: This research is supposed by the Public Technology Research Project of Zhejiang Province (LGG18F030008).

Conflicts of Interest: The authors declare no conflicts of interests.

\section{Abbreviations}

$\begin{array}{ll}\text { GRA } & \text { Grey relational analysis } \\ \text { INSs } & \text { Interval neutrosophic sets } \\ \text { MADM } & \text { Multi-attribute decision-making } \\ \text { MCGDM } & \text { Multi-criteria group decision making } \\ \text { MVNSs } & \text { Multi-valued neutrosophic sets } \\ \text { NCHWAGA } & \text { Neutrosophic cubic hybrid weighted arithmetic and geometric aggregation } \\ \text { NCSs } & \text { Neutrosophic cubic sets } \\ \text { NCWAA } & \text { Neutrosophic cubic weighted arithmetic average } \\ \text { NCWGA } & \text { Neutrosophic cubic geometric weighted average } \\ \text { SNSs } & \text { Simplified neutrosophic sets } \\ \text { SVNSs } & \text { Single-valued neutrosophic sets } \\ \text { WAA } & \text { Weighted arithmetic average } \\ \text { WGA } & \text { Geometric average }\end{array}$

\section{References}

1. Zadeh, L.A. The concept of a linguistic variable and its application to approximate reasoning Part I. Inf. Sci. 1975, 8, 199-249. [CrossRef]

2. Atanassov, K.T. Intuitionstic fuzzy sets. Fuzzy Sets Syst. 1986, 20, 87-96. [CrossRef]

3. Smarandache, F. Neutrosophy: Neutrosophic Probability, Set, and Logic; American Research Press: Rehoboth, DE, USA, 1998.

4. Wang, H.; Smarandache, F.; Zhang, Y.Q.; Sunderraman, R. Interval Neutrosophic Sets and Logic: Theory and Applications in Computing; Hexis: Phoenix, AZ, USA, 2005.

5. Wang, H.; Smarandache, F.; Zhang, Y.Q.; Sunderraman, R. Single valued neutrosophic sets. Multisp. Multistruct. 2010, 4, 410-413.

6. Ye, J. A multicriteria decision-making method using aggregation operators for simplified neutrosophic sets. J. Intell. Fuzzy Syst. 2014, 26, 2459-2466. [CrossRef]

7. Wang, J.Q.; Li, X.E. An application of the TODIM method with multi-valued neutrosophic set. Control Decis. 2015, 30, 1139-1142.

8. Peng, J.J.; Wang, J.Q. Multi-valued neutrosophic sets and its application in multi-criteria decision-making problems. Neutrosophic Sets Syst. 2015, 10, 3-17. [CrossRef]

9. Liu, P.D.; Tang, G.L. Some power generalized aggregation operators based on the interval neutrosophic numbers and their application to decision making. J. Intell. Fuzzy Syst. 2016, 30, 2517-2528. [CrossRef]

10. Ye, J. Improved cosine similarity measures of simplified neutrosophic sets for medical diagnoses. Artif. Intell. Med. 2015, 63, 171-179. [CrossRef]

11. Ye, J.; Fu, J. Multi-period medical diagnosis method using a single valued neutrosophic similarity measure based on tangent function. Comput. Methods Programs Biomed. 2016, 123, 142-149. [CrossRef]

12. Shi, L. Correlation Coefficient of simplified neutrosophic sets for bearing fault diagnosis. Shock Vib. 2016. [CrossRef] 
13. Ye, J. Single valued neutrosophic similarity measures based on cotangent function and their application in the fault diagnosis of steam turbine. Soft Comput. 2017, 21, 817-825. [CrossRef]

14. Smarandache, F. n-Valued refined neutrosophic logic and its applications in physics. Prog. Phys. 2013, 4, 143-146.

15. Broumi, S.; Smarandache, F. Intuitionistic neutrosophic soft set. J. Inf. Comput. Sci. 2013, 8, 130-140.

16. Ye, J. Multiple-attribute decision-making method under a single-valued neutrosophic hesitant fuzzy environment. J. Intell. Fuzzy Syst. 2015, 24, 23-36. [CrossRef]

17. Broum, S.; Smarandache, F.; Dhar, M. Rough neutrosophic sets. Neutrosophic Sets Syst. 2014, 3, $62-67$.

18. Ali, M.; Deli, I.; Smarandache, F. The theory of neutrosophic cubic sets and their applications in pattern recognition. J. Intell. Fuzzy Syst. 2016, 30, 1957-1963. [CrossRef]

19. Jun, Y.B.; Smarandache, F.; Kim, C.S. Neutrosophic cubic sets. New Math. Nat. Comput. 2017, 13, 41-45. [CrossRef]

20. Lu, Z.; Ye, J. Cosine measures of neutrosophic cubic sets for multiple attribute decision-making. Symmetry 2017, 9, 121. [CrossRef]

21. Banerjee, D.; Giri, B.C.; Pramanik, S.; Smarandache, F. GRA for multi attribute decision making in neutrosophic cubic set environment. Neutrosophic Sets Syst. 2017, 15, 64-73.

22. Pramanik, S.; Dalapati, S.; Alam, S.; Roy, T.K.; Smarandache, F. Neutrosophic cubic MCGDM method based on similarity measure. Neutrosophic Sets Syst. 2017, 16, 44-56.

23. $\mathrm{Xu}, \mathrm{Z}$.S. A note on linguistic hybrid arithmetic averaging operator in multiple attribute group decision making with linguistic information. Group Decis. Negot. 2006, 15, 593-604. [CrossRef]

24. Liu, P. Multiple attribute decision-making methods based on normal intuitionistic fuzzy interaction aggregation operators. Symmetry 2017, 9, 261. [CrossRef]

25. Liu, P.; Wang, P. Some improved linguistic intuitionistic fuzzy aggregation operators and their applications to multiple-attribute decision making. Int. J. Inf. Technol. Decis. Mak. 2017, 16, 817-850. [CrossRef]

26. Wei, G.W.; Alsaadi, F.E.; Hayat, T.; Alsaedi, A. Picture 2-tuple linguistic aggregation operators in multiple attribute decision making. Soft Comput. 2018, 22, 989-1002. [CrossRef]

27. Wei, G.W. Pythagorean fuzzy interaction aggregation operators and their application to multiple attribute decision making. J. Intell. Fuzzy Syst. 2017, 33, 2119-2132. [CrossRef]

28. Luo, X.; Xu, Z.; Gou, X. Exponential operational laws and new aggregation operators of intuitionistic Fuzzy information based on Archimedean T-conorm and T-norm. Int. J. Mach. Learn. Cybern. 2018, 9, 1261-1269. [CrossRef]

29. Wang, J.; Tian, C.; Zhang, X.; Zhang, H.; Wang, T. Multi-criteria decision-making method based on simplified neutrosophic linguistic information with cloud model. Symmetry 2018, 10, 197. [CrossRef]

30. Shi, L.; Ye, J. Dombi aggregation operators of neutrosophic cubic sets for multiple attribute decision-making. Algorithms 2018, 11, 29. [CrossRef]

31. Zhan, J.; Khan, M.; Gulistan, M.; Ali, A. Applications of neutrosophic cubic sets in multi-criteria decision making. Int. J. Uncertain. Quantif. 2017, 7, 377-394. [CrossRef]

32. Jun, Y.B.; Smarandache, F.; Kim, C.S. P-union and P-intersection of neutrosophic cubic sets. St. Univ. Ovidius Constanta 2017, 25, 99-115. [CrossRef]

33. Ye, J. Linguistic neutrosophic cubic numbers and their multiple attribute decision-making method. Information 2017, 8, 110. [CrossRef]

34. Ye, J. Intuitionistic fuzzy hybrid arithmetic and geometric aggregation operators for the decision-making of mechanical design schemes. Appl. Intell. 2017, 47, 743-751. [CrossRef]

35. Lu, Z.; Ye, J. Single-valued neutrosophic hybrid arithmetic and geometric aggregation pperators and their decision-making method. Information 2017, 8, 84. [CrossRef]

36. Smarandache, F. Neutrosophic Overset, Neutrosophic Underset, and Neutrosophic Offset. Similarly for Neutrosophic Over-/Under-/Off-Logic, Probability, and Statistics. Pons Editions: Bruxelles, Belgique, 2016. Available online: https: / arxiv.org/ftp/arxiv/papers/1607/1607.00234.pdf (accessed on 4 January 2019).

(C) 2019 by the authors. Licensee MDPI, Basel, Switzerland. This article is an open access article distributed under the terms and conditions of the Creative Commons Attribution (CC BY) license (http:/ / creativecommons.org/licenses/by/4.0/). 